\title{
Nanomechanical characterization by double-pass force-distance mapping
}

\author{
Yavuz S Dagdas ${ }^{1}$, M Necip Aslan ${ }^{2}$, Ayse B Tekinay ${ }^{1}$, \\ Mustafa O Guler ${ }^{1}$ and Aykutlu Dâna ${ }^{1}$ \\ ${ }^{1}$ UNAM Institute of Materials Science and Nanotechnology, Bilkent University, \\ 06800 Ankara, Turkey \\ ${ }^{2}$ Department of Physics, Istanbul Technical University, Istanbul, Turkey \\ E-mail: aykutlu@unam.bilkent.edu.tr
}

Received 29 December 2010, in final form 10 May 2011

Published 15 June 2011

Online at stacks.iop.org/Nano/22/295704

\begin{abstract}
We demonstrate high speed force-distance mapping using a double-pass scheme. The topography is measured in tapping mode in the first pass and this information is used in the second pass to move the tip over the sample. In the second pass, the cantilever dither signal is turned off and the sample is vibrated. Rapid (few $\mathrm{kHz}$ frequency) force-distance curves can be recorded with small peak interaction force, and can be processed into an image. Such a double-pass measurement eliminates the need for feedback during force-distance measurements. The method is demonstrated on self-assembled peptidic nanofibers.

(Some figures in this article are in colour only in the electronic version)
\end{abstract}

\section{Introduction}

Nanomechanical characterization attracts attention in a number of fields, including semiconductor process characterization, materials science, polymer science and biology [1-3, 7]. Typically, force-distance curves are analyzed using a tipsample interaction model of choice to extract the material stiffness and adhesion properties [4-6]. Apart from traditional force-distance measurements, several techniques such as pulsed force mode or microsecond force spectroscopy have been used to obtain complete nanomechanical maps of surfaces. Acquisition of force-distance maps using minimal indentation forces allows the extraction of mechanical properties of fragile features such as biomolecules. Rapid acquisition also allows greater throughput and reduced susceptibility to mechanical drifts. Existing techniques for nanomechanical mapping generally require special cantilevers or special hardware for data collection.

In this paper we describe a simple method for nanomechanical mapping using a double-pass scheme. Double-pass measurements typically use the tapping mode to record the topography, and the cantilever is lifted a known amount in the second pass to measure long range interactions. Traditionally, double-pass imaging has been used to characterize electrostatic and magnetic forces [8-10]. In this study, topography and phase information is recorded in tapping mode in the first pass and the cantilever dither is turned off in the second pass, while vibrating the sample with a small amplitude at a frequency much lower than the cantilever resonance frequency (figure 1). At each oscillation cycle of the sample, the cantilever comes into contact with the surface, and a force-distance map (FDM) is recorded by auxiliary electronics (such as a digital oscilloscope or a data acquisition board). The FDM can be then analyzed to extract material properties.

\section{Experimental details}

In the demonstration of the technique, we use a commercial atomic force microscope (AFM, Asylum Research MFP3D) with built-in double-pass imaging capability. A piezo bimorph disc element (a buzzer) is glued onto a polycarbonate plate with a circular opening that closely matches the piezo diameter. The assembly can be fixed on the flexure scanner of the AFM system using magnets. A silicon sample is glued on to the buzzer. The sample area can be as large as a square centimeter. Such an assembly can be constructed using low-cost and widely available components and is disposable. Alternatively, the cantilever position can be modulated through the displacement mechanism that provides $z$-direction feedback of the AFM. We prefer to modulate 


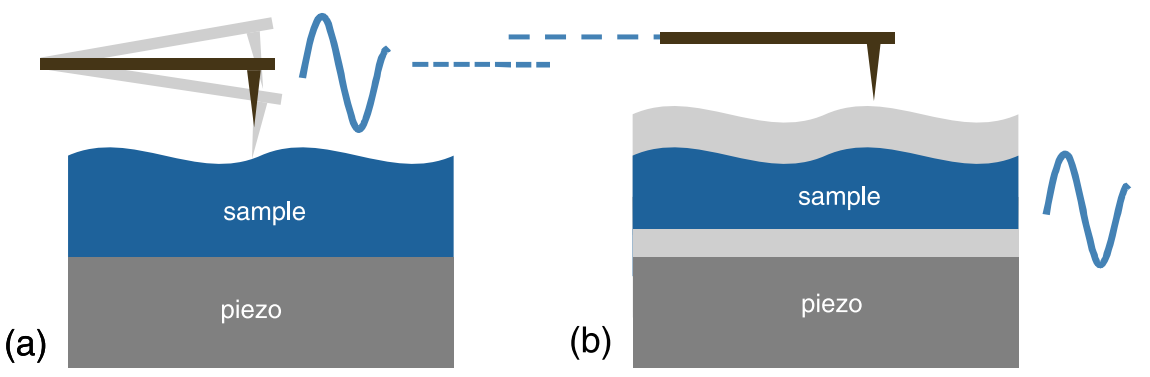

Figure 1. (a) The topography of the sample is acquired in the tapping mode by vibrating the cantilever, and (b) the topographic information is used in the second pass to keep the tip-sample separation constant, while vibrating the sample and recording the force-distance curves. The force-distance curves can then be used to extract the tip-sample interaction parameters.
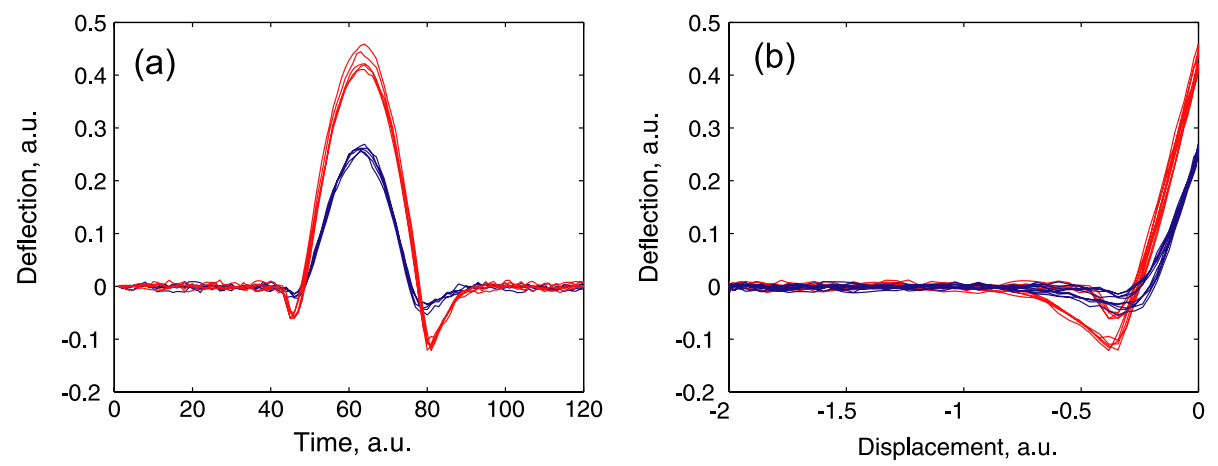

Figure 2. (a) In the second pass the time-dependent cantilever deflection is recorded and (b) by fitting a sinusoidal oscillation to the tip-sample separation, the deflection-time curve is converted into a deflection-displacement curve. Two representative locations on silicon (red, higher peak deflection) and organic layers (blue, lower peak deflection) are shown.

the tip-sample separation by the bimorph piezo disc as this method does not require interfacing to the system electronics. The output of the AFM system is used to generate a signal to trigger an external signal generator (Stanford Research Systems DS345). The sinusoidal output of the external signal generator drives the piezo disc for a set duration, enabling force-distance mapping during the second pass. The deflection signal is low pass filtered with a $100 \mathrm{kHz}$ bandwidth and captured using a data acquisition board.

In the imaging and spectroscopy experiments, cantilevers of spring constant 3-40 $\mathrm{N} \mathrm{m}^{-1}$ with resonant frequencies of $f_{0} \sim 60-400 \mathrm{kHz}$ are used (provided by BudgetSensors). Softer cantilevers allow reduced tip-sample forces; however, post-snap-off oscillations are observed in the force-distance curves. High stiffness cantilevers $\left(k>10 \mathrm{~N} \mathrm{~m}^{-1}\right)$ display reduced snapping and produce better elasticity contrast, at the expense of increased tip-sample forces. In the experiments, the sample is typically oscillated at a frequency of $f_{\mathrm{s}}=$ $2 \mathrm{kHz}$, allowing FDMs to be acquired at about $2 \mathrm{~Hz}$ line frequency. The oscillation of the sample in the FDM part of the measurement is stable and has constant amplitude. In such a case, the tip-sample distance can be assumed to be a sinusoid with $f_{\mathrm{s}}=2 \mathrm{kHz}$ frequency. At the point of closest approach, or the peak of the sinusoid, tip-sample contact takes place, and the cantilever is deflected due to the attractive and repulsive forces (figure 2(a)). The time-dependent deflection data are then converted to a deflection-distance graph as shown in figure 2(b). The slope of the deflection-displacement
(DD) curve can be understood in terms of the relative spring constants of the cantilever and the tip-sample effective spring constant. The DD curve forms the basis of the force-distance (FD) curve, where the deflection variable is converted into the force variable simply by multiplying by the cantilever's spring constant. The slope of the DD curve can be related to the cantilever's spring constant and tip radius using the DMT model [11]. The calculation results given in figures 3(a) and (b) show the dependence of the slope of the DD curves on sample elasticity for given spring constants and tip radii (for a peak force of about $10 \mathrm{nN}$ ). The slope of the deflection-distance curve can be approximated by $[12,13]$

$$
\frac{\delta \text { Deflection }}{\delta \text { Displacement }}=\frac{\sqrt[3]{6 R_{\mathrm{tip}} F E^{2}}}{k_{\mathrm{c}}+\sqrt[3]{6 R_{\mathrm{tip}} F E^{2}}}
$$

where $k_{\mathrm{c}}$ is the cantilever spring constant, $R_{\text {tip }}$ is the tip radius, $E$ is the sample elastic modulus (with an infinitely hard tip) and $F$ is the pressing force. The above equation agrees well with the calculation shown in figure 3 for $F \simeq 10 \mathrm{nN}$ pressing force. It must be noted, however, that equation (1) requires the repulsive contact force for quantitative estimation, and without accounting for the attractive portion of the FD (or DD) curve, a reliable estimation cannot be made. In our scheme, the full FD (or DD) curve is measured, and the attractive forces can properly be accounted for. In order to get a quantitative correspondence between the slope and the sample elasticity, both the spring constant and the tip radius must 


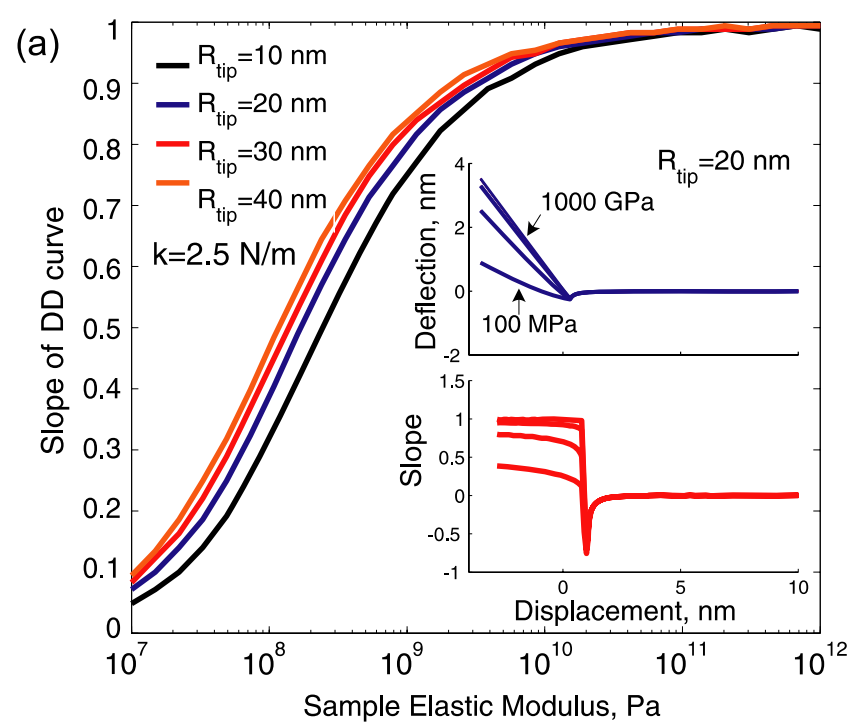

(b)

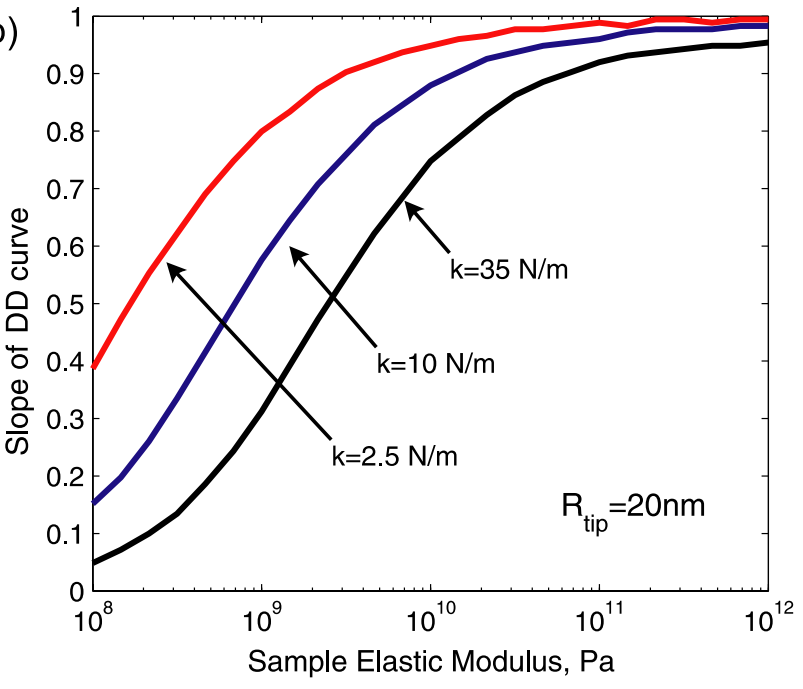

Figure 3. (a) Using the Derjaguin-Muller-Toporov interaction model deflection-displacement (DD) curves are calculated for a spring constant of $2.5 \mathrm{~N} \mathrm{~m}^{-1}$ for various tip radii, as a function of sample elasticity. Peak values of the slope are plotted against sample elasticity. The insets show actual calculated deflection-displacement curves and their slopes as a function of tip displacement. (b) The calculations are repeated for a tip radius of $20 \mathrm{~nm}$, for various common spring constants. Softer cantilevers are more suitable for soft materials and large spring constants are suitable for materials with large elastic modulus.

be calibrated. The spring constant calibration can be carried out in a number of different ways $[14,15]$. In our system, the piezo stages are closed-loop controlled. The calibration of $z$-displacement has been previously carried out and due to the presence of low-drift displacement monitors on the $z$ stage (linear variable differential transformer sensors), the $z$ displacement serves as a reliable reference. The cantilever deflection is first calibrated using an approach curve where the cantilever is oscillated near its fundamental resonance and the tip-sample separation is gradually decreased. The approach curve is used to extract amplitude-to-voltage and deflectionto-voltage conversion factors. The Brownian spectrum is then used to estimate the resonance frequency, quality factor (a)

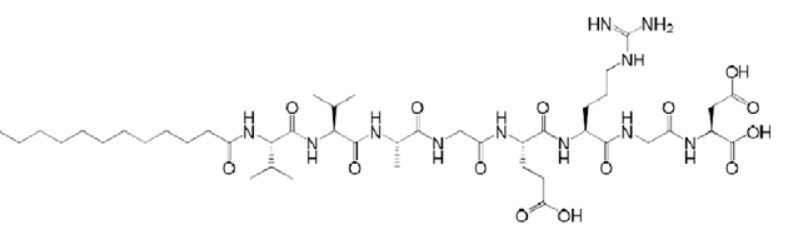

(b)

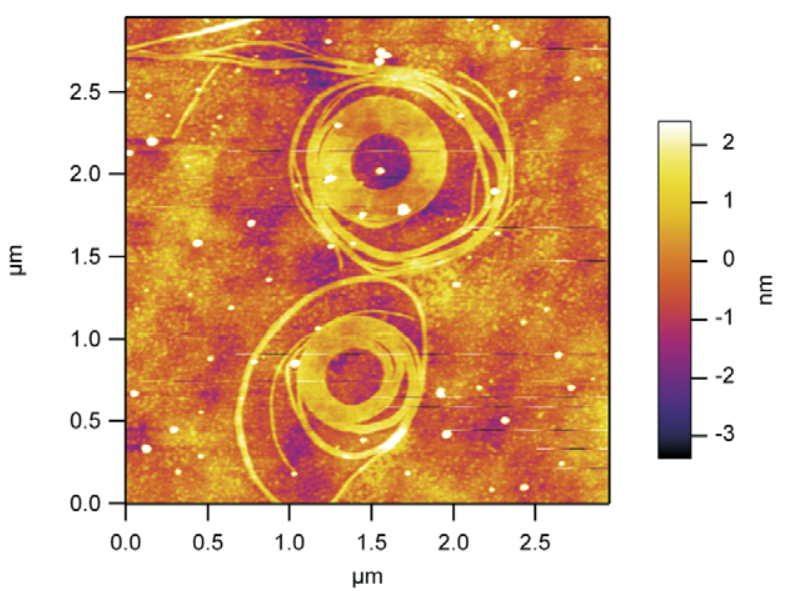

Figure 4. (a) Schematic representation of amphiphile peptides forming the nanofibers. (b) Typical flattened topography image of secondary structures formed by self-assembled nanofibers on a silicon surface.

and spring constant of the cantilever. The tip radius cannot be calibrated by using the cantilever signals alone, and DD curves on soft and hard reference samples must be collected to estimate the tip radius. Ideally, a patterned polymer layer such as PMMA on silicon should be used to estimate the tip radius. It is seen from theoretical calculations (figures 3(a) and (b)) that an uncertainty in the tip radius will produce a smaller error bar on soft materials than on harder materials. Also by using a stiffer cantilever, the uncertainty can be decreased for materials with large elastic modulus (figure 3(b)).

The sample used in the experiments consists of selfassembled peptide amphiphile (PA) nanofibers, with a typical diameter of 2-10 nm, dropcast on silicon. The PA molecule used in this study is composed of an alkyl tail, $\beta$ sheet forming valine-valine-alanine-glycine (VVAG) peptide sequence followed by a glutamic acid residue, which is effective in increasing the solubility of the molecule, and a bioactive epitope arginine-glycine-aspartic acid (RGD), a peptide sequence that enhances cell adhesion (figure 4(a)) [16]. PA molecules self-assemble into fiber networks through the addition of a divalent cation, $\mathrm{Ca}^{2+}$, and $\mathrm{pH}$ change. The resulting fibers assemble into secondary structures on the surface in the form of thin layers (figure 4(b)). Such thin layers consist of one or a few layers of fibers and are suitable for imaging using the AFM. Details of synthesis and sample preparation can be found elsewhere [17].

\section{Results and discussion}

Typical results acquired with a cantilever of spring constant $k \simeq 2.5 \mathrm{~N} \mathrm{~m}^{-1}$ and resonance frequency $\left(f_{0} \simeq 75 \mathrm{kHz}\right)$ are given in figure 5(a) which shows the unprocessed topography. 
(a)

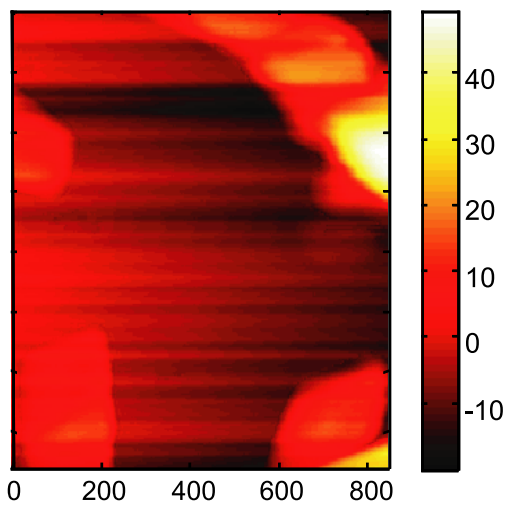

(c)

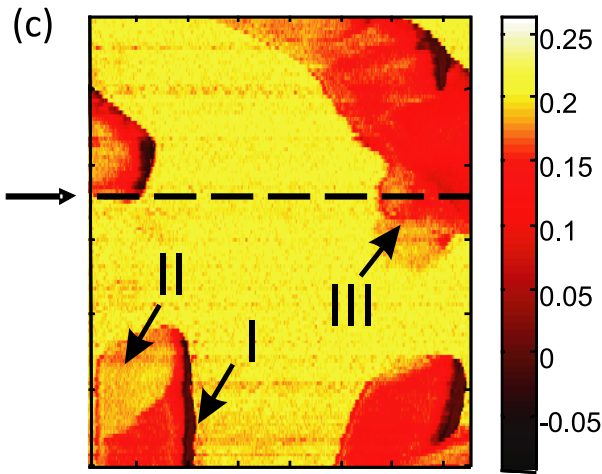

(b)

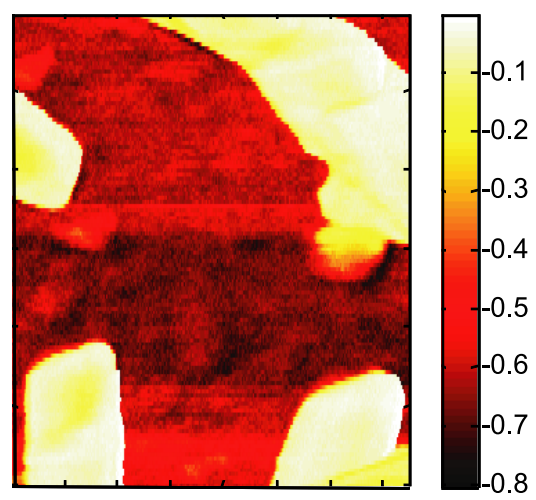

(d)

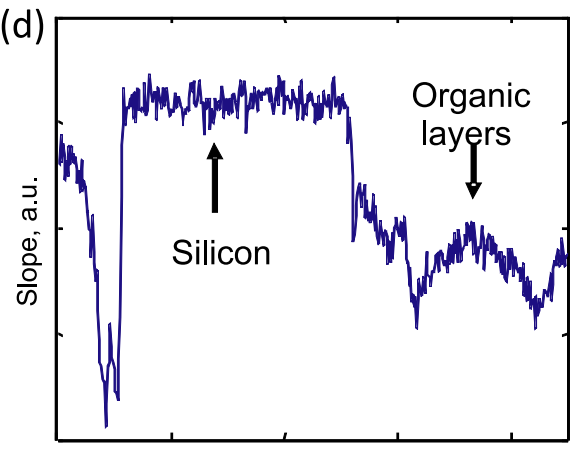

Distance, a.u.

Figure 5. (a) For double-pass force-distance mapping, in the first pass the topography is acquired using the tapping mode (820 $\mathrm{nm}$ wide image, $45 \mathrm{~nm}$ full-color scale). (b) The adhesion map can be extracted from the force-distance maps obtained in the second pass (arbitrary units). (c) The slope of the force-distance map gives elasticity related information (arbitrary units). The slope is simply calculated by using the peak deflection and minimum deflection during approach. This simple calculation is susceptible to topography measurement errors due to finite feedback speed, as well as attractive/repulsive regime bistability at step edges (arrow I). Thin portions of the organic layers appear to be stiffer due to compression of the film beyond the film thickness during contact (arrows II and III). (d) A line section of the slope data shows repeatability on flat topography.

Here, the data are not corrected by flattening and show the thermal drift during the topographic imaging. Typical parameters used in the imaging are $20 \mathrm{~nm}$ tapping amplitude, $75 \%$ amplitude set point, driven slightly below the resonance frequency to favor repulsive mode imaging.

The adhesion map (figure 5(b)) is extracted by plotting the maximum negative deflection during retraction of the curve shown in figure 2(b). Instead of explicitly expressing the elasticity, the extracted slope of the deflection-distance curves is shown in figure 5(c). Quantitative sample elasticity can be inferred from the slope using figure 3. The extracted slope value is repeatable and a section is shown in figure $5(\mathrm{~d})$. It is seen that, despite significant thermal drifts (more than a few nanometers in $z$ ) during the course of imaging (figure 5(a)), both adhesion (figure 5(b)) and elasticity (figure 5(c)) show insensitivity to the much smaller drift (about $0.2 \mathrm{~nm}$ ) between topography and force-map acquisition. It is also seen that, although the organic components of the sample are very fragile, satisfactory results can be obtained and tapping-mode imaging can be sustained throughout the image. The section shows the excellent repeatability of the slope measurement on flat topography. It must be noted that the slope extraction algorithm used in figure 5(c) is not sophisticated, causing surface tracking errors, and attractive/repulsive regime bistabilities during topography acquisition (which are manifested in the amplitude and phase images, data not shown) couple into the elasticity channel at step edges (figure 5(c), arrow I). In forcedistance measurements, typically the DMT interaction model is used to estimate the sample elasticity. This model is known to produce reliable quantitative results on films much thicker than the indentation depth; however, it fails for thin suspended or partially suspended layers [18] or for thin layers (with thickness smaller than the indentation depth) of soft material on a hard substrate. This is demonstrated in our measurements, where a greater apparent elastic modulus is observed for thin portions of the organic layers (figure 5(c), arrows II and III). The contact portion of the DMT model is based on Hertzian contact of a sphere on a flat surface. In reality, geometry also plays an important role in the contact forces and the contact model has to be corrected in a non-flat sample topography with features on the order of the tip radius.

We also show histograms of slope (figure 6(a)) and adhesion (figure 6(b)) extracted from the data of figures 5(b) and (c). The slope histograms show two distinct domains with slopes peaking at around 0.93 (silicon substrate) and 0.62 (organic overlayers). Based on the calculations shown in figure 3(a), using the spring constant of $k=2.5 \mathrm{~N} \mathrm{~m}^{-1}$ and assuming a nominal tip radius of $20 \mathrm{~nm}$, the organic 

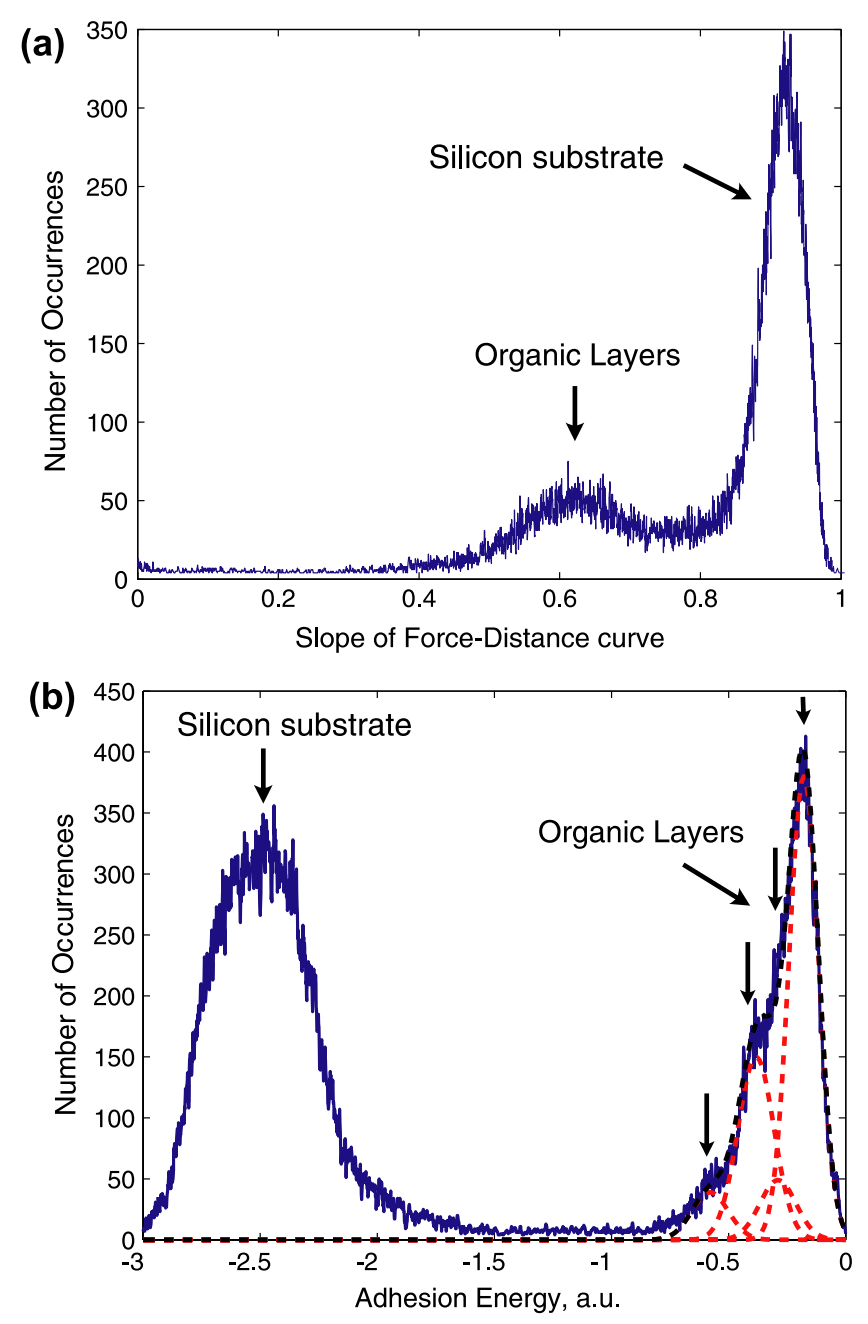

Figure 6. (a) Histograms of the slope of the deflection-distance curves extracted from the deflection-distance data shown in figure 5(c) are shown. Two distinct domains with slopes peaking at around 0.93 (silicon substrate) and 0.62 (organic overlayers) are identifiable. Based on the calculations shown in figure 3(a), the organic layers have an elastic modulus of about $0.3 \mathrm{GPa}$. (b) The adhesion histograms also show two distinct domains, the silicon substrate producing more adhesion and the organic layers producing less adhesion. The organic layer portion of the histogram shows distinct detaching events (downward pointing arrows with corresponding Gaussian fits), possibly due to debuckling of self-assembled fibers or bond breaking.

layers are found to have an elastic modulus of about $0.3 \mathrm{GPa}$. The deflection-displacement curve on the silicon substrate is expected to have a slope close to 1 as opposed to 0.93 . We attribute this to errors in the slope calibration process. Nevertheless, the measurements demonstrate that the method can be used for quantitative analysis. The adhesion histograms also show two distinct domains, the silicon substrate producing more adhesion and the organic layers producing less adhesion. Interestingly, the organic layer portion of the histogram shows distinct detaching events (downward pointing arrows with corresponding Gaussian fits), possibly due to debuckling of self-assembled fibers or breaking of bonds within the layer or between the organic layer and the tip. Without further experiments specifically designed to distinguish between different mechanisms, the exact origin of the steps in the adhesion histogram cannot be identified.

\section{Conclusion}

In conclusion, we demonstrate a simple method for acquiring force-distance maps, which can find application in a variety of fields such as polymer science and biology. Sample drift, especially in the $z$-direction, causes an increase or decrease in the peak forces during force-distance mapping; however, it is seen that, despite the presence of sample drifts or tapping-mode imaging artifacts, force-distance mapping can produce satisfactory results even on fragile molecular layers. It is also demonstrated in our measurements that the DMT model fails to accurately describe the contact of a spherical tip with a thin soft planar layer on a hard substrate. Therefore, we note that, irrespective of the technique used to measure the tip-sample interaction curves, the effect of sample geometry is an important factor that has to be properly modeled in nanomechanical property mapping experiments using the AFM.

\section{Acknowledgment}

This work was partially funded by TUBITAK 107T547, by the State Planning Agency of the Turkish Republic Project UNAM.

\section{References}

[1] Jalili N and Laxminarayana K 2004 Mechatronics 14 907-45

[2] Zhang H, Grim P C M, Vosch T, Wiesler U-M, Berresheim A J, Mllen K and De Schryver F C 2000 Langmuir 169294

[3] Zhu M, Akari S and Mohwald H 2001 Nano Lett. 1 569-73

[4] Sahin O, Yaralioglu G, Grow R, Zappe S F, Atalar A, Quate C and Solgaard O 2004 Sensors Actuators A 114183

[5] Sahin O, Magonov S, Su C, Quate C F and Solgaard O 2007 Nat. Nanotechnol. 2507

[6] Dong M, Husale S and Sahin O 2009 Nat. Nanotechnol. 4514

[7] Yaralioglu G G, Degertekin F L, Crozier K B and Quate C F 2000 J. Appl. Phys. 8774916

[8] Jacobs H O, Knapp H F, Mueller S and Stemmer A 1997 Ultramicroscopy $6939-49$

[9] Proksch R, Skidmore G D, Dahlberg E D, Foss S, Schmidt J J, Merton C, Walsh B and Dugas M 1996 Appl. Phys. Lett. 692559

[10] Winkler A, Mhl T, Menzel S, Kozhuharova-Koseva R, Hampel S, Leonhardt A and Bchner B J 2006 J. Appl. Phys. 99104905

[11] Derjaguin B V, Muller V M and Toporov Y P 1975 J. Colloid Interface Sci. 53 314-25

[12] Overney R M, Meyer E, Frommer J, Guntherodt H J, Fujihara M, Takano H and Goto Y 1994 Langmuir 10 1281-6

[13] Akhremitchev B B, Brown H G, Graner S R and Walker G C 2001 Microsc. Microanal. 7 32-8

[14] Cleveland J P, Manne S, Bocek D and Hansma P K 1993 Rev. Sci. Instrum. 64 403-5

[15] Hutter J L and Bechhoefer J 1993 Rev. Sci. Instrum. 64 1868-73

[16] Pierschbacher M D and Ruoslahti E 1984 Nature 309 30-3

[17] Dagdas Y S, Tombuloglu A, Tekinay A B, Dana A and Guler M O 2011 Soft Matter 73524

[18] Sarioglu A F, Atalar A and Degertekin F L 2004 Appl. Phys. Lett. 845368 